\title{
THE THIRD GALACTIC CARBON SYMBIOTIC
}

\author{
Regina E. Schulte-Ladbeck ${ }^{1, *}$, D. Jack MacConnell ${ }^{2,+}$ and Nelson Zarate ${ }^{3}$ \\ ${ }^{1}$ Washburn Observatory, University of Wisconsin-Madison, 475 North Charter Street, \\ Madison, Wisconsin 53706 \\ ${ }^{2}$ Astronomy Programs, Computer Sciences Corporation, Space Telescope Science \\ Institute, Homewood Campus, Baltimore, Maryland 21218 \\ ${ }^{3}$ Space Telescope Science Institute, Homewood Campus, Baltimore, Maryland 21218
}

\begin{abstract}
Of the more than 100 symbiotic stars now known in our Galaxy, only two have previously been reported to contain carbon stars as their cool components. We here present observations of a third such object, which we wish to call Weaver's star since Weaver (1972) first identified it as a symbiotic.
\end{abstract}

Weaver's star has the equatorial coordinates (1950) $\alpha=12^{\mathrm{h}} 48^{\mathrm{m}} \mathrm{m}_{21} \mathrm{~s}_{9}, \delta=-64^{\circ} 43^{\prime} 48^{\prime \prime}$ and the galactic coordinates $1=303 \stackrel{\circ}{\circ} .0, \mathrm{~b}=-2^{\circ} .2$. These coordinates put the object in the Coalsack region. The spectrum was reported by Weaver to contain a very strong $\mathrm{H}_{\alpha}$ emission line, weaker $\mathrm{H}_{\beta}$, and progressively weaker series members until H9, prominent [OIII] $\lambda \lambda 4363 \AA, 4959 \AA$ and $5007 \AA$ and a $\mathrm{He}$ II $\lambda 4686 \AA$ line almost as strong as $\mathrm{H}_{\beta}$. The continuum was noted as being medium strong at $\mathrm{H}_{\alpha}$ and comparatively fainter in the blue region of the spectrum. However, no absorption bands are mentioned. The photographic magnitudes as observed by Weaver between May 19 and May 29, 1971 did not show significant variability. The values given are $U=14.6, B=14.3, V=13.8$, and $\mathrm{I}_{\mathrm{ph}}=12.0$, with an internal p.e. of $0 \mathrm{~m}_{07}$. Weaver's star is included in the various Allen catalogs (e.g. Allen 1984). Allen lists the star under the name SS 38 (Sanduleak and Stephenson 1973) as a D-type of variable $K$ magnitude $(5.7<K<6.5)$.

In Figure 1 we present a Reticon spectrum of Weaver's star along with a spectrum of the bright and well known galactic carbon symbiotic UV Aur. Both spectra cover the same far red wavelength region from $6700 \AA<\lambda<9600 \AA$. The characteristic absorption bands of $\mathrm{CN}$ are strong in the spectrum of UV Aur and can be easily recognized in the spectrum of Weaver's star, albeit at a lower signal to noise level. We attempted to classify Weaver's star on Richer's system (Richer 1971) by comparing it with UV Aur which Richer assigns to C9 II at minimum light. His criteria are that the absence of the $\mathrm{Ca}$ II triplet $(\lambda \lambda 8498 \AA, 8542 \AA$ and $8662 \AA)$ indicates a type later than C7, whereas a weak K I doublet $(\lambda \lambda 7665 \AA, 7699 \AA)$ favours a type close to C9; both criteria apply to our spectra of Weaver's star.

\footnotetext{
${ }^{*}$ Based on observations collected at the European Southem Observatory, La Silla, Chile.

+ Visiting Astronomer, Cerro Tololo Interamerican Observatory, National Optical Astronomy Observatories, operated by the Association of Universities for Research in Astronomy, Inc., under contract with the U.S. National Science Foundation.
} 
We have subsequently taken a number of red and blue spectra of Weaver's star during the past year. A large number of strong emission lines is present, as is typical for D-type symbiotics. The ratio of $\mathrm{H}_{\alpha}$ to $\mathrm{H}_{\beta}$ is large, 16:1, indicating a large amount of extinction near or on the path to the star. Weaver's star is also listed by Allen as a radio source. We have also found a detection of this object in the IRAS point source catalog.

The discovery of a new galactic carbon symbiotic has important implications for the evolution of symbiotic binaries as well as for the statistics of carbon stars. Weaver's star is the first galactic $D$-type carbon symbiotic and, showing variable $\mathrm{K}$ magnitudes, it is perhaps the first galactic symbiotic carbon Mira. Other carbon symbiotics have been located in the Large and Small Magellanic Clouds and the Draco dwarf spheroidal galaxy, respectively. A more detailed discussion will be given elsewhere.

ACKNOWLEDGEMENTS. R.E.S.-L. was supported by the Deutsche Forschungsgemeinschaft. D.J.M. thanks W.P.Bidelman and C.B.Stephenson for calling to his attention the positional coincidence of Weaver's star with that of a C star in an unpublished list of D.J.M.

\section{REFERENCES}

Allen, D.A.: 1984, Proc.A.S.A: 5, 369

Richer, H.B.: 1971, Astrophys.J. 167, 521

Sanduleak, N. and Stephenson, C.B.: 1973, Astrophys.J. 185, 899

Weaver, W.B.: 1972, Publ.astron.Soc.Pacific 84, 854

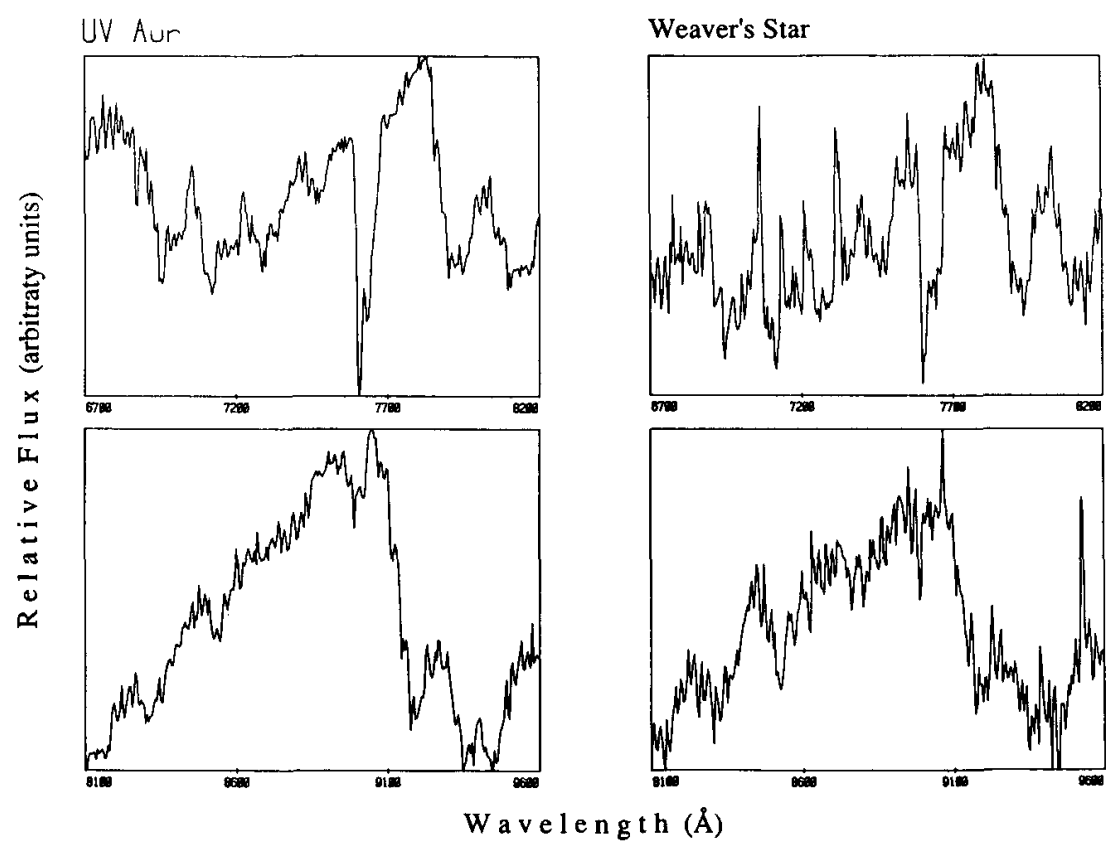

Figure 1. The far red spectrum of the well known galactic carbon symbiotic UV Aur shows the strong $\mathrm{CN}$ bands that are typical for carbon stars. The same bands are now identified in Weaver's symbiotic star. Together with UV Aur and UKS Ce-1, Weaver's star is the third carbon symbiotic known in our Galaxy. 